\title{
Computation of Wavelet and Multiwavelet Transforms Using Fast Fourier Transform
}

\author{
W. A. Mahmoud \\ Professor in. College of Engineering, Uruk University. Baghdad. Iraq.
}

Profwaleed54@gmail.com

\begin{abstract}
A novel fast and efficient algorithm was proposed that uses the Fast Fourier Transform (FFT) as a tool to compute the Discrete Wavelet Transform (DWT) and Discrete Multiwavelet Transform. The Haar Wavelet Transform and the GHM system are shown to be a special case of the proposed algorithm, where the discrete linear convolution will adapt to achieve the desired approximation and detail coefficients. Assuming that no intermediate coefficients are canceled and no approximations are made, the algorithm will give the exact solution. Hence the proposed algorithm provides an efficient complexity verses accuracy tradeoff. The main advantages of the proposed algorithm is that high band and the low band coefficients can be exploited for several classes of signals resulting in very low computation.
\end{abstract}

Keywords: Discrete Wavelet Transform; Haar Wavelet Transform; Fast Fourier Transform; Multiwavelet Transform.

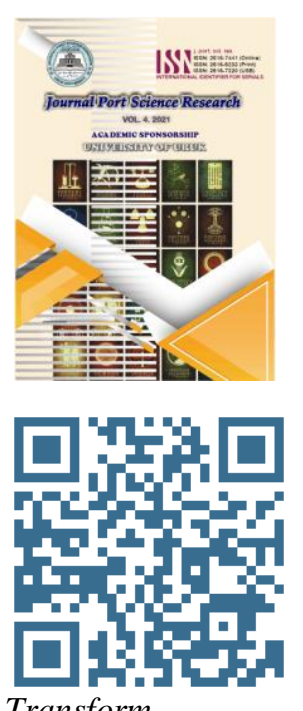

\section{INTRODUCTION}

In 19th century, the French mathematician, J. Fourier, showed that any periodic function can be expressed as an infinite sum of periodic complex exponential functions. Many years after this remarkable property of periodic functions was discovered, the ideas were generalized to nonperiodic functions, and then to periodic or non-periodic discrete time signals. After that, Fourier transform (FT) became a very famous tool for computer calculations . [2,1] Note that in the Fourier Transform equation, the integration is from minus infinity to plus infinity over time. So, no matter when the component with frequency appears in time, it will affect the result of the integration equally as well. The lack of time information in the spectrum is one serious weakness of Fourier Transform. That is why Fourier transform is not suitable if the signal has time varying frequency, i.e., the nonstationary signals.[3]

To solve the above problem, the Windowed Fourier Transform is used. The basic idea is to divide the signal into small enough segments, where these segments can be assumed to be stationary. The width of this window must be equal to the segment of the signal where this assumption is valid.

The Windowed Fourier Transform has several problems. Using a window of infinite length will result in Fourier Transform with good frequency resolution, but no time information. On the other hand, in order to obtain a stationary sample, a small enough window is required in which the signal is stationary. The narrower is the window, the better is the time resolution, and better the assumption of stationary, but the poorer the frequency resolution. However, the Wavelet transform solves the dilemma of resolution to a certain extent [4]. Notice that a wavelet at a scale occupies only part of the length of the signal being analyzed. To cover the full length, additional wavelets have to be added and, at level 3, there are 23=8 wavelets at equally spaced intervals along the horizontal axis.

\section{WAVELET EQUATIONS}

The discrete wavelet transform (DWT) operates on a data vector whose length is an integer power of 2 , transforming it into a numerically different vector of the same length. Like the Discrete Fourier Transform (DFT), the wavelet transform is invertible and in fact orthogonal, the inverse transform, when viewed as a big matrix, is simply the transpose of the transform. Both DFT and DWT, therefore, can be viewed as a rotation in function space, from the input space (or time) domain. The basis functions are the unit vectors ei, or Dirac delta functions in the continuum limit, to a different domain. For the DFT, this new domain has basis functions that are the familiar sines and cosines. In the wavelet domain, the basis functions are somewhat more complicated and have the fanciful names mother functions and wavelets.[5]

Wavelet functions generated from one single function $\psi$, which is called mother wavelet, by the dilation, the factor a and the translation factor $b$ : 
$\varphi_{a, b}(x)=\vdots a:^{-\frac{1}{2}} \varphi\left(\frac{x-b}{a}\right) \quad \ldots$

The fundamental idea behind wavelets is to analyze the signal at different scales or resolutions, which is called multiresolution analysis. Wavelets are a class of functions used to localize a given signal in both space and scaling domains. A family of wavelets can be constructed from a mother wavelet. Compared to Windowed Fourier analysis, a mother wavelet is stretched or compressed to change the size of the window. In this way, big wavelets give an approximate image of the signal, while smaller and smaller wavelets zoom in on details. Therefore, wavelets automatically adapt to both the highfrequency and the low-frequency components of a signal by different sizes of windows. Any small change in the wavelet representation produces a correspondingly small change in the original signal, which means local mistakes will not influence the entire transform. The wavelet transform is suited for non-stationary signals (signals with interesting components at different scales) [6].

For best performance in some applications, wavelet transforms require filters that combine a number of desirable properties, such as orthogonally and symmetry. However, the design possibilities for wavelets are limited because they cannot simultaneously possess all these desirable properties [7]. The relatively new field of Multiwavelets shows promise in removing some of the limitations of wavelets [8].

\section{WAVELET THEORY}

Wavelets are functions that satisfy certain requirements. The very name wavelet comes from the requirement that they should integrate to zero, "waving" above and below the $\mathrm{x}$ axis. The diminutive connotation of wavelet suggests the function has to be well localized. Other requirements are technical and need mostly to insure quick and easy calculations of direct and inverse wavelet transform [6].

Wavelet novices often search for the reason of not using traditional Fourier methods. There are some important differences between Fourier analysis and wavelets. Fourier basis functions are localized in frequency but not in time. Small frequency changes in the Fourier transform will produce changes everywhere in the time domain. Wavelets are local in both frequency/scale (via dilations) and in time (via translations). This localization is an advantage in many cases [8].

Second, many classes of functions can be represented by wavelets in a more compact way. For example, functions with discontinuities and functions with sharp spikes usually take substantially fewer wavelet basis functions than sine-cosine basis functions to achieve a comparable approximation. This sparse coding makes wavelet excellent tools in data compression [9].

Wavelet analysis provides an alternative way of breaking the signal down into its constituent parts [4]. The shapes of the components of the decomposed signal depend on the shape of the analyzing wavelet. There are an infinite number of possibilities for this, but only a small subset of these meets the conditions that are necessary if the wavelets are to give an accurate decomposition and also be orthogonal to each other. In other words, the analyzing wavelet determines the shape of the building blocks from which the signal $\mathrm{f}$ is constructed (the basis functions into which the signal $\mathrm{f}$ can be decomposed) [3]. For Haar Wavelet with bases functions Fig. (1) Gives the values of these functions $h(n)$ and $g(n)$, and three levels of decompositions. The implementation of Wavelet equations are illustrated in Fig. (1). In this figure, three levels of decomposition are depicted. $h$ and $g$ are low-pass and highpass filters corresponding to the coefficients respectively. The down-pointing arrows denote a decimation or downsampling by two.

$h[n]=\left[\frac{-\sqrt{2}}{2}, \frac{\sqrt{2}}{2}\right] g[n]=\left[\frac{\sqrt{2}}{2}, \frac{\sqrt{2}}{2}\right]$

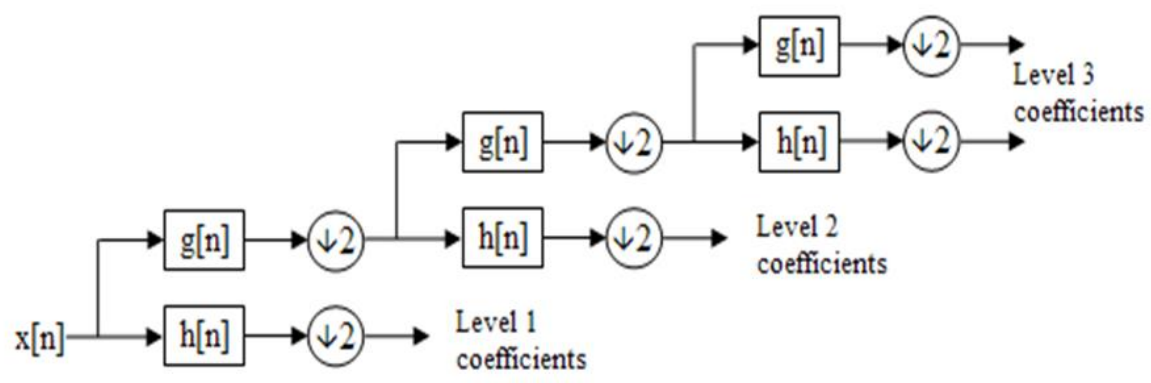

Fig. (1) Three level Haar Wavelet Decompositions

The following steps can view the computation of 1-D single level DWT:

1) Compute the linear convolution between the input $x$ and the filters $h \& g$.

2) Apply Decimation by 2 by taking only the odd sequence values of the results of the linear convolution. 
3) Compute the linear convolution between the a's and the filters $h \& g$ for more than one level of decomposition.

The resultant a's and b's are the Wavelet Coefficients.
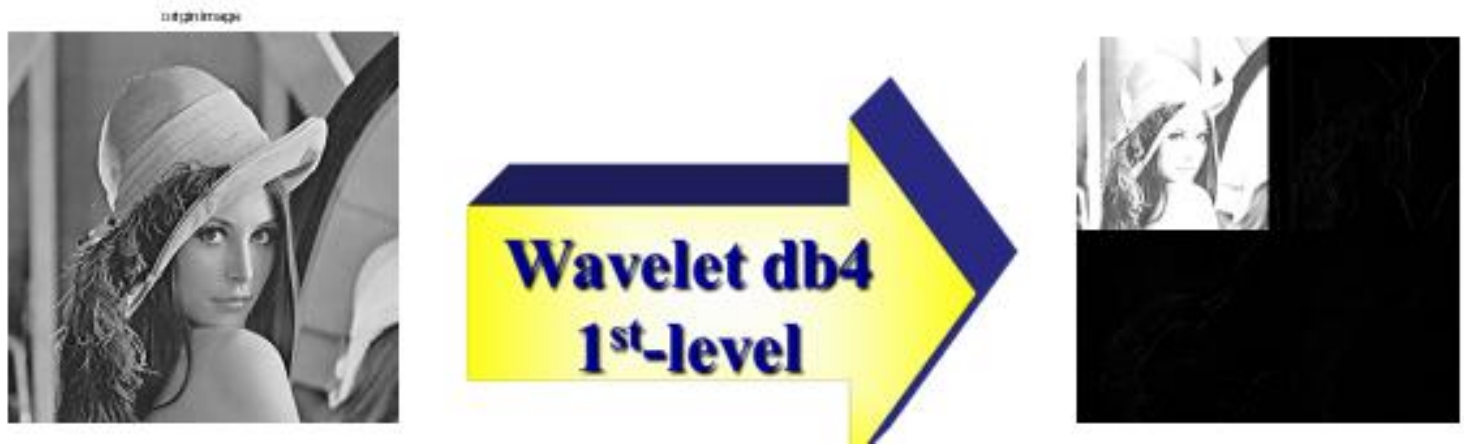

Fig. (2) Wavelet Decompositions of an image.

\section{DISCRETE FOURIER TRANSFORM (DFT)}

A Fourier Transform will break apart a time signal and will return information about the frequency of all sine waves needed to simulate that time signal. It converts a sequence of $\mathrm{N}$ equally spaced real or complex sample $\mathrm{x} 0$ $\mathrm{x} 1$....etc, of a function $\mathrm{x}(\mathrm{t})$ of time (or another variable, depending on the application) into a sequence of $\mathrm{N}$ complex numbers xk by the following summation:
Let $W_{N}=e^{-j 2 \pi / N}$, hence

$X(k)=\sum_{n=0}^{N-1} x_{n} W_{N}^{n k}$

With the numbers $X_{k}$ known, the inverse DFT exactly recovers the sample values $x_{n}$ through the following summation

$$
\begin{aligned}
& \left.x(n)=\frac{1}{N} \sum_{k=0}^{N-1} X(k) W_{N}^{-n k} \ldots . .3\right) \\
& {\left[\begin{array}{l}
X(0) \\
X(1) \\
X(2) \\
X(3)
\end{array}\right]=\left|\begin{array}{cccc}
1 & 1 & 1 & 1 \\
1 & W_{4}^{1} & W_{4}^{2} & W_{4}^{3} \\
1 & W_{4}^{2} & W_{4}^{4} & W_{4}^{6} \\
1 & W_{4}^{3} & W_{4}^{6} & W_{4}^{9}
\end{array}\right| *\left[\begin{array}{l}
x(0) \\
x(1) \\
x(2) \\
x(3)
\end{array}\right]}
\end{aligned}
$$

Decimation-In-Time FFT Algorithm is simplified in Fig. (2).

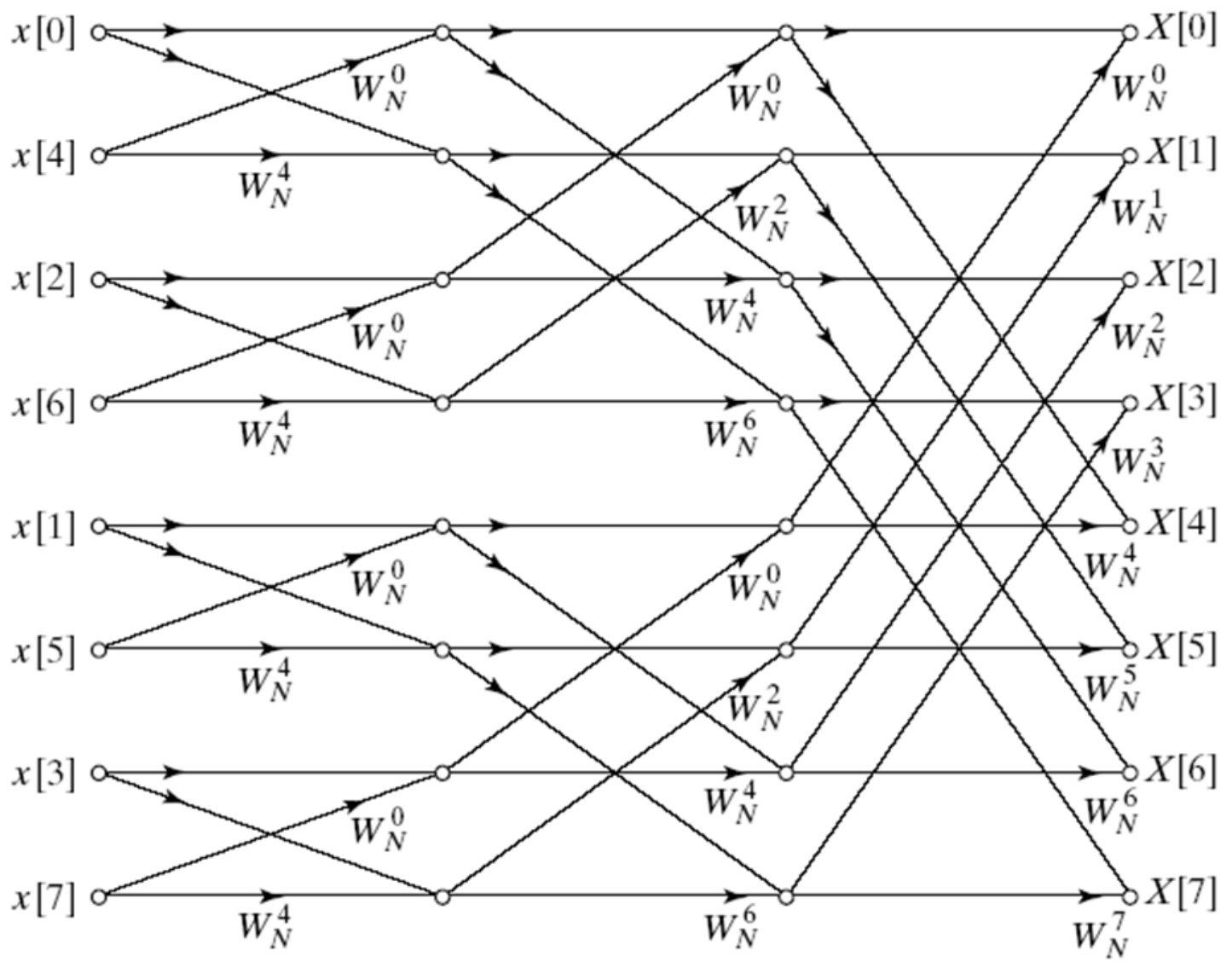

Fig. (3) Flow graph for an 8-points DIT-FFT algorithm. 
The Fourier matrix GF can be expressed in terms of the separated matrix SM [4]:

$\mathrm{SM}=\left[\begin{array}{llll}1 & 0 & 0 & 0 \\ 0 & 0 & 1 & 0 \\ 0 & 1 & 0 & 0 \\ 0 & 0 & 0 & 1\end{array}\right]$

$G F_{N}=G F_{N} S M_{N}^{T} S M_{N}$

$G F_{N}=\left[\begin{array}{cc}I_{N / 2} & D_{N / 2} \\ I_{N / 2} & -D_{N / 2}\end{array}\right]\left[\begin{array}{cc}G F_{N / 2} & 0 \\ 0 & G F_{N / 2}\end{array}\right] S M_{N}$

Where $\mathrm{D}_{\overline{\mathrm{N}}}$ is diagonal matrix with $\mathrm{w}_{\mathrm{N}}^{\mathrm{i}}$ on the diagonal.

\section{THE PROPOSED METHOD OF COMPUTING DWT USING FFT:}

Concerning with discrete wavelet analysis and the signal to be analysed is assumed to have been sampled at equally spaced intervals. The shapes of the components of the decomposed signal depend on the shape of the analysing wavelet. There are an infinite number of possibilities for this, but only a small subset of these meets the conditions that are necessary if the wavelets are to give an accurate decomposition and also be orthogonal to each other. In other words, the analysing wavelet determines the shape of the building blocks from which the signal $\mathrm{f}$ is constructed (the basis functions into which the signal $\mathrm{f}$ can be decomposed). The longer the original sequence, which must be a power of 2 , the more levels there are in the transform. For a given time duration, increasing the number of sampling points increases the amount of detail available.

The discrete wavelet transform (DWT) operates on a data vector whose length is an integer power of 2 , transforming it into a numerically different vector of the same length. Like the Discrete Fourier Transform (DFT), the wavelet transform is invertible and in fact orthogonal, the inverse transform, when viewed as a big matrix, is simply the transpose of the transform. Both DFT and DWT, therefore, can be viewed as a rotation in function space, from the input space (or time) domain. The basis functions are the unit vectors ei, or Dirac delta functions in the continuum limit, to a different domain. For the DFT, this new domain has basis functions that are the familiar sines and cosines. In the wavelet domain, the basis functions are somewhat more complicated and have the fanciful names mother functions and wavelets.

A particular set of wavelets is specified by a particular set of numbers, called wavelet filter coefficients. Here, we will largely restrict ourselves to wavelet filters in a class discovered by Daubechies. This class includes members ranging from highly localized to highly smooth. The simplest (and most localized) member, often called DAUB4 or D4, has only four coefficients, $\mathrm{c} 0, \ldots, \mathrm{c} 3$. For the moment we specialize to this case for ease of notation.

The calculation of Haar Wavelet transform can be expressed in matrix representation $\llbracket \mathrm{WH} \rrbracket \_\mathrm{N}$ in the following form:

$$
\begin{aligned}
\mathrm{WH}_{\mathrm{N}} & =1 / \sqrt{2}\left[\begin{array}{cccc}
1 & 1 & 0 & 0 \\
0 & 0 & 1 & 1 \\
1 & -1 & 0 & 0 \\
0 & 0 & 1 & -1
\end{array}\right] \\
\mathrm{WH}_{\mathrm{N}} & =\left[\begin{array}{llll}
\mathrm{W}_{\mathrm{N} / 4} & & \\
& \mathrm{I}_{\mathrm{N} / 4} & \\
& & \mathrm{I}_{\mathrm{N} / 4}
\end{array}\right]\left[\begin{array}{lll}
\mathrm{W}_{\mathrm{N} / 4} & \\
& \mathrm{I}_{\mathrm{N} / 4}
\end{array}\right]
\end{aligned}
$$

It can be shown that due to the orthogonality of the bases functions that:

$$
\begin{aligned}
& \mathrm{I}_{\mathrm{N}}=\mathrm{WH}_{\mathrm{N}}^{\mathrm{T}} \mathrm{WH}_{\mathrm{N}} \quad \ldots(10) \\
& \mathrm{WH}_{\mathrm{N}}=\mathrm{WH}_{\mathrm{N}} \mathrm{GF}_{\mathrm{N}}^{\mathrm{T}} \mathrm{GF}_{\mathrm{N}} \mathrm{SM}_{\mathrm{N}}^{\mathrm{T}} \mathrm{SM}_{\mathrm{N}}
\end{aligned}
$$

Hence it can be seen that the Wavelet transform can be computed using FFT using the above equation (11). This can be expressed in terms of following equation:

$\mathrm{WH}_{\mathrm{N}}=\left[\begin{array}{cc}\mathrm{J}_{\mathrm{N} / 2} & \mathrm{~K}_{\mathrm{N} / 2} \\ \mathrm{~L}_{\mathrm{N} / 2} & \mathrm{M}_{\mathrm{N} / 2}\end{array}\right]\left[\begin{array}{cc}\mathrm{GF}_{\mathrm{N} / 2} & 0 \\ 0 & \mathrm{GF}_{\mathrm{N} / 2}\end{array}\right] \mathrm{SM}_{\mathrm{N}} \ldots$

Where, $J_{N / 2}, K_{N / 2}, L_{\frac{N}{2}}$ and $M_{N / 2}$ are all diagonal matrices.

This splitting, filtering and decimation can be repeated on the scaling coefficients to give the two-scale structure. The first stage of two banks divided the spectrum of into a lowpass and high-pass band, resulting in the scaling coefficients and wavelet coefficients at lower scale. The second stage then divides that low-pass band into another lower low-pass band and a band-pass band and so on. Equation (12) can be summarized for the computation of the discrete wavelet transform (DWT) using FFT through the following proposed algorithm:

Step -1- computes the FFT of the input signal $(x(n))$ which gives $\mathrm{X}(\mathrm{k})$.

Step -2- computes the FFT of the $g$ filter which results in $\mathrm{G}(\mathrm{k})$.

Step -3- compute the FFT of the h filter which produce $\mathrm{H}(\mathrm{k})$. Step -4- Obtain the dot product between $\mathrm{X}(\mathrm{k})$ and $\mathrm{G}(\mathrm{k})$ to get $\mathrm{GX}(\mathrm{k})$.

Step -5- Obtain the dot product between $\mathrm{X}(\mathrm{k})$ and $\mathrm{H}(\mathrm{k})$ to get $\mathrm{HX}(\mathrm{k})$.

Step -6- Calculate the low band Wavelet coefficient after taking the inverse FFT of GX(k).

Step -7- Calculate the high band Wavelet coefficient after taking the inverse FFT of $\mathrm{HX}(\mathrm{k})$.

Fig. (4) Shows a demonstrated example on the above procedure applied to the signal $x(n)=[1,-1,2,1]$. After taking the FFT of the signal and the high pass filter of the wavelet transform $h(n)=[-1,1,0,0]$, the multiplication of the results was performed. Next, the inverse FFT of the result of the multiplication was achieved. The Decimation of the results gives the high band values of $H=[2,1]$.

Fig. (5) Demonstrate the algorithm to obtain the approximation coefficients for the same input function which results in $\mathrm{G}=[2,1$ 


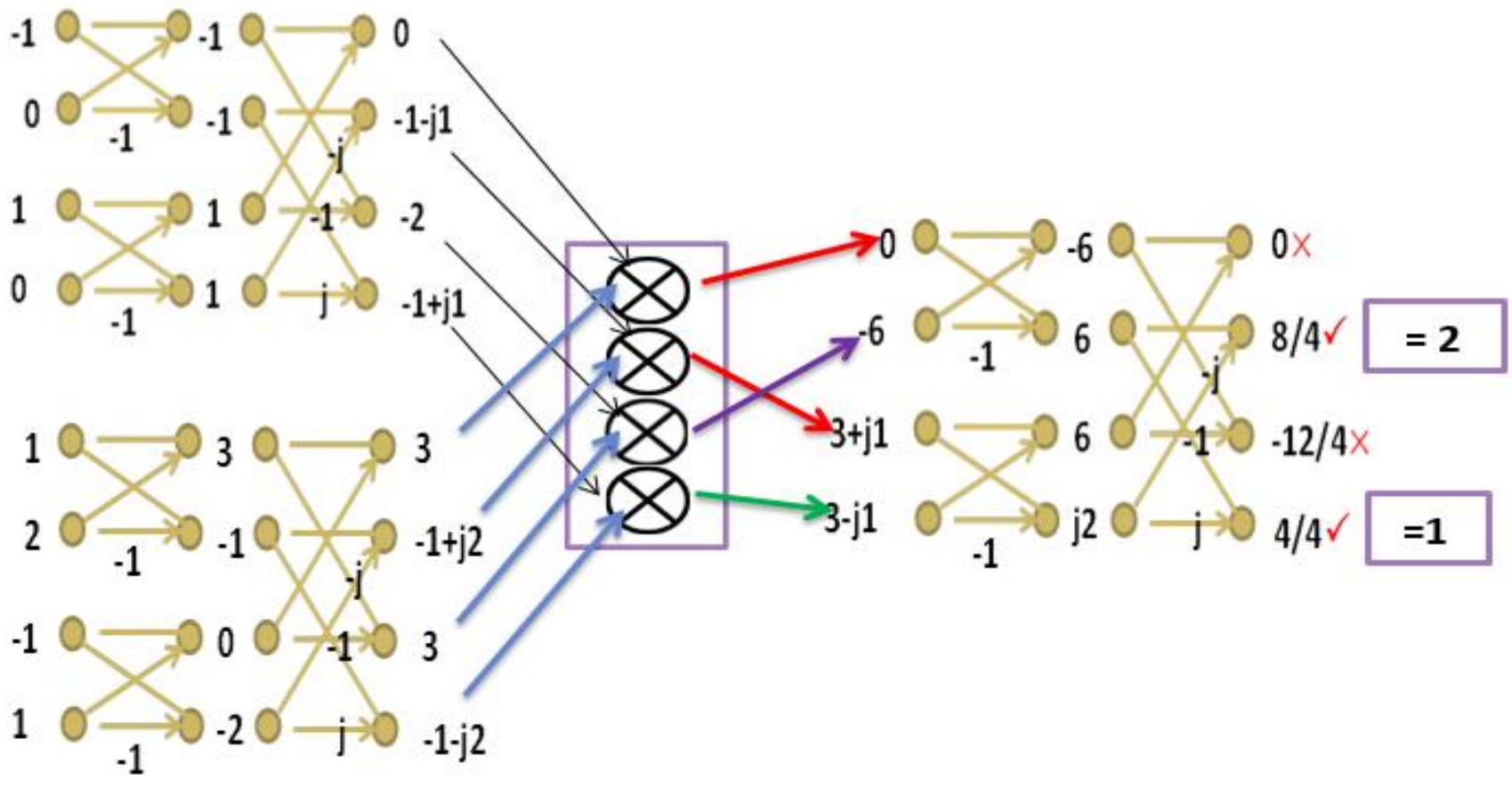

Fig (4) Computation of the DWT Detail Coefficients

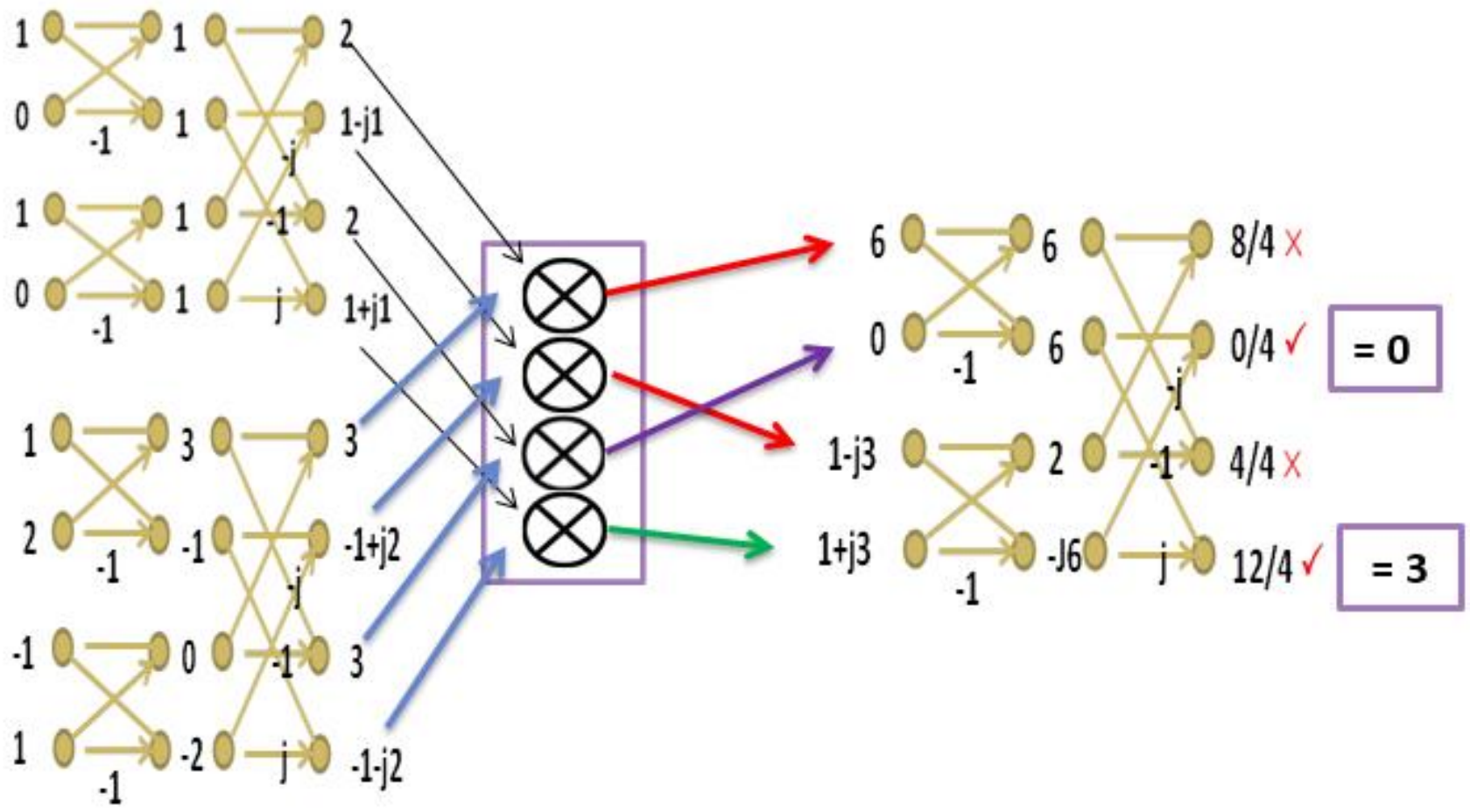

Fig (5) Computation of the DWT approximation Coefficients 

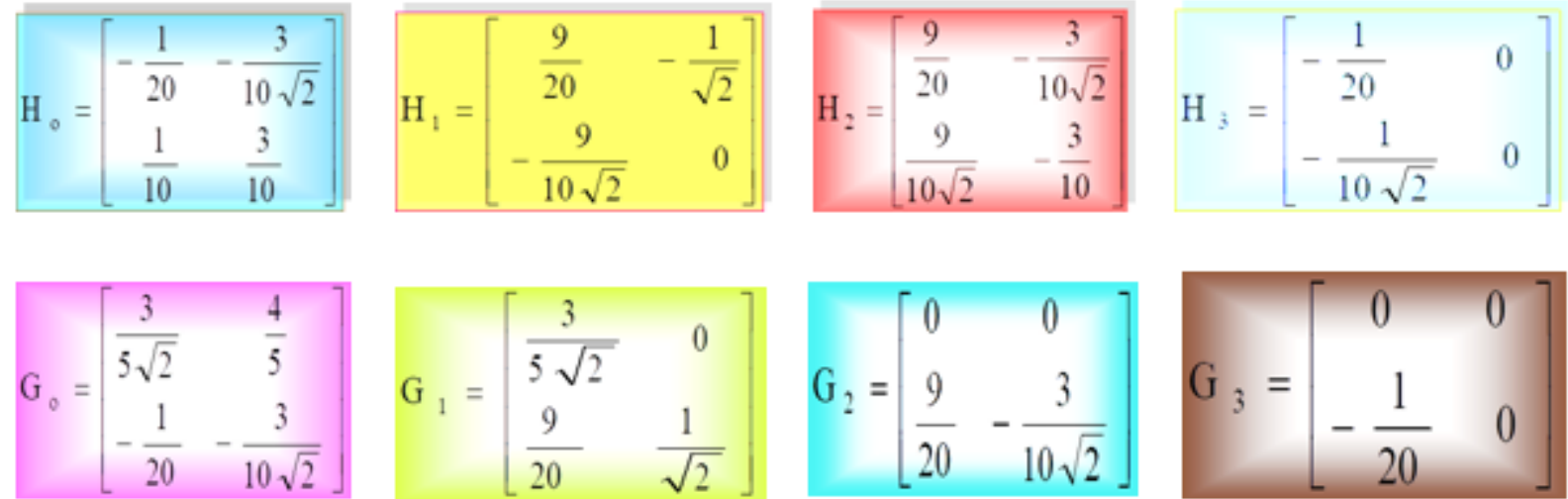

Fig. (6) The values of the orthogonal matrices of Multiwavelet transform.

The algorithm for the computation of DMWT using FFT algorithm summarized in the following steps which are the same as for Wavelet transform:

Step -1- computes the FFT of the input signal $(\mathrm{x}(\mathrm{n}))$ which gives $\mathrm{X}(\mathrm{k})$.

Step -2- computes the FFT of the $g$ filters which results in $\mathrm{G} 0(\mathrm{k}), \ldots, \mathrm{G} 3(\mathrm{~K})$.

Step -3- compute the FFT of the $h$ filters which produce $\mathrm{H} 0(\mathrm{k}), \ldots, \mathrm{H} 3(\mathrm{~K})$.

Step -4- Obtain the dot product between $\mathrm{X}(\mathrm{k})$ and $\mathrm{G} 0(\mathrm{k}), \ldots$, $\mathrm{G} 3(\mathrm{~K})$ to get $\mathrm{GX}(\mathrm{k})$.

Step -5- Obtain the dot product between $\mathrm{X}(\mathrm{k})$ and $\mathrm{H} 0(\mathrm{k}), \ldots$, $\mathrm{H} 3(\mathrm{~K})$. to get $\mathrm{HX}(\mathrm{k})$.

Step -6- Calculate the low band Wavelet coefficient after taking the inverse FFT of $\mathrm{GX}(\mathrm{k})$ and $\mathrm{HX}(\mathrm{k})$. .

Step -7- Calculate the high band Wavelet coefficient after taking the inverse FFT of $\mathrm{HX}(\mathrm{k})$.

\section{CONCLUSIONS}

The proposed algorithm for the computation of DWT and DMWT using the FFT proposed here have the following characteristics:

1) It was shown that the proposed method computes the exact results.

2) Due to the implementing of FFT in its realization its computation complexity is on the same order of that for the FFT i.e. $(\mathrm{O}(\log 2 \mathrm{~N}))$.

3) The signals are made spares that can be used to speed up the proposed algorithm through the dropping of the insignificant data.

4) Using section pruning of the algorithm that corresponds to the significant twiddle factors, the magnitudes will be decreased those results in speeding up the proposed algorithm.

5) 5- The algorithm is very efficient and can result in a building denoising capability which can be used in several applications in future for this purpose.

\section{REFERENCES}

[1] Wang, Z., Wan, F., Wong, C. M., \& Zhang, L. (2016). Adaptive Fourier decomposition based ECG denoising. Computers in Biology and Medicine, 77, 195-205. https://doi.org/10.1016/j.compbiomed.2016.08.013

[2] Martis, R. J., Acharya, U. R., \& Min, L. C. (2013). ECG beat classification using PCA, LDA, ICA and Discrete Wavelet Transform. Biomedical Signal Processing and Control, 8(5), 437-448. https://doi.org/10.1016/j.bspc.2013.01.005

[3] Yu, B., \& Zhang, Y. (2012). A simple method for predicting transmembrane proteins based on wavelet transform. International Journal of Biological Sciences, 9(1), 22-33. https://doi.org/10.7150/ijbs.5371

[4] Guo, H., \& Burrus, C. S. (1996). Fast approximate Fourier transform via wavelets transform. In Wavelet Applications in Signal and Image Processing IV (Vol. 2825, pp. 250-259). SPIE. https://doi.org/10.1117/12.255236

[5] Tse, N. C. F., Chan, J. Y. C., Lau, W. H., \& Lai, L. L. (2012). Hybrid wavelet and hilbert transform with frequencyshifting decomposition for power quality analysis. IEEE Transactions on Instrumentation and Measurement, 61(12), 3225-3233. https://doi.org/10.1109/TIM.2012.2211474

[6] Zhang, Z., Telesford, Q. K., Giusti, C., Lim, K. O., \& Bassett, D. S. (2016). Choosing wavelet methods, filters, and lengths for functional brain network construction. PLOS ONE, 11(6). https://doi.org/10.1371/journal.pone.0157243 
[7] Singh, R., Rajpal, N., \& Mehta, R. (2020). Wavelet and kernel dimensional reduction on arrhythmia classification of ECG signals. EAI Endorsed Transactions on Scalable Information Systems, 7(26), 1-13. https://doi.org/10.4108/eai.137-2018.163095

[8] Fowler, J. E., \& Hua, L. (2002). Wavelet transforms for vector fields using omnidirectionally balanced multiwavelets. IEEE Transactions on Signal Processing, 50(12), 3018-3027. https://doi.org/10.1109/TSP.2002.805488

[9] Mallat, S. (2009). A Wavelet Tour of Signal Processing. A Wavelet Tour of Signal Processing. Elsevier Inc. https://doi.org/10.1016/B978-0-12-374370-1.X0001-8

[10] Proceedings of the 1998 IEEE-SP International Symposium on Time-Frequency and Time-Scale Analysis. (1998). Proceedings of the IEEE-SP International Symposium on Time-Frequency and Time-Scale Analysis. IEEE.

[11]Xia, X. G. (1998). A new prefilter design for discrete multiwavelet transforms. IEEE Transactions on Signal Processing, 46(6), 1558-1570. https://doi.org/10.1109/78.678469

[12]S, Y. (2018). Denoising of ECG Signals using Multiwavelet Transform. HELIX, 8(1), 2696-2700. https://doi.org/10.29042/2018-2696-2700

[13] Paulchamy, B., Chidambaram, S., \& Basheer, J. M. (2021). Removal of Artifacts from Electroenchaphalography Signal using Multiwavelet Transform. In Journal of Physics: Conference Series (Vol. 1921). IOP Publishing Ltd. https://doi.org/10.1088/1742-6596/1921/1/012008

[14] Alramahi N, Bush M, Swash MR (2018) Retracted: Numerically- analysed multiwavelet transform computations: multidimensional compression case studies. Journal of Fundamental and Applied Sciences. 10(S4), PP691-693. https://www.ajol.info/index.php/ifas/article/view/171952

[15] Attakitmongcol, K., Hardin, D. P., \& Mitchell Wilkes, D. (2001). Multiwavelet prefilters - Part II: Optimal orthogonal prefilters. IEEE Transactions on Image Processing, 10(10), 1476-1487. https://doi.org/10.1109/83.951534

[16] Strela, V., \& Walden, A. T. (1998). Orthogonal and biorthogonal multiwavelets for signal denoising and image compression. In Wavelet Applications V (Vol. 3391, pp. 96-107). SPIE. https://doi.org/10.1117/12.304924.

[17] Mahmoud, W. A. (2011), A Smart Single Matrix Realization of Fast Walidlet Transform. International Journal of Research and Reviews in Computer Science, 1(2). https://www.researchgate.net/publication/323906455 